\title{
Comparative Analysis of Anatomy Following Intraoperative Cholangiograms in Elective Laparoscopic Cholecystectomies
}

\author{
Hosam Elghannam, b, c, Vikram Malik ${ }^{\mathrm{a}, \mathrm{b}}$, Hasib Bhojwani ${ }^{\mathrm{a}, \mathrm{b}}$
}

\begin{abstract}
Background: Laparoscopic cholecystectomies are performed more than 650,000 times per year in the United States. The importance of understanding the anatomy of the biliary system variants is essential to all surgical procedures, which can minimize surgical errors and common bile duct (CBD) injuries. Anatomic variants are seen in 18$39 \%$ of cholecystectomies, wherein 3-6\% lead to increased risk of biliary tract injuries. We aim to show that anatomic variants do exist at a substantial rate and that performing intraoperative cholangiograms can prevent associated complications.
\end{abstract}

Methods: Data on 22 elective laparoscopic cholecystectomies due to acute cholecystitis were collected. All received intraoperative cholangiograms utilizing standard angiographic catheterization. Intraoperative cholangiograms were evaluated for anatomic variants. Complications were noted to compare and document relative risk of each anatomic variant.

Results: Thirty-six percent of patients presented with anatomic variations in their biliary system, right posterior segmental duct (RPSD) draining into the $\mathrm{CBD}$, trifurcation of right anterior segmental duct (RASD), RPSD, and left hepatic duct (LHD) draining into the CBD, and accessory hepatic duct draining into common hepatic duct; all occurred at the same frequency of $10.5 \%$ each respectively, with the remaining $5.26 \%$ coming from patients with the RPSD draining into the LHD. Patients with anatomic variations had zero intraoperative complication.

Conclusion: This study highlights the importance of intraoperative cholangiograms in understanding the anatomy of the biliary system, and minimizing bile duct injuries to prevent the morbid risk associated with the procedure for those patients with variations in ductal anatomy.

Keywords: Intraoperative cholangiogram; Gallbladder; Cholecystectomy; Anatomy; Biliary system

Manuscript accepted for publication October 02, 2015

${ }^{a}$ Windsor University School of Medicine, St. Kitts and Nevis ${ }^{b} 10050$ Westpark Drive, Unit 1510, Houston, TX 77042, USA

${ }^{\mathrm{c}}$ Corresponding Author: Hosam Elghannam, 10050 Westpark Drive Unit 1510, Houston, TX 77042, USA. Email: HosamAmr@me.com

doi: http://dx.doi.org/10.14740/jcs280w

\section{Introduction}

Laparoscopic cholecystectomy is one of the most commonly performed procedures in the United States, with more than 650,000 procedures being done each year [1]. The gallbladder is an ovoid shaped sac, which stores bile, adjacent to the liver. The gallbladder is comprised of a fundus, body, and an infundibulum, which drains by the cystic duct. The cystic duct then joins the common hepatic duct (CHD) to form the common bile duct (CBD) and drains into the duodenum. These make up what is called the biliary tree [2].

Intraoperative cholangiograms are a valuable tool for a surgeon. They allow for the detection of common duct stone, delineation of the anatomy of the bile ducts, and the identifications of abnormalities such as cysts and/or tumors [2].

Intraoperative cholangiograms prevent the surgeons from misidentifying the CBD as the cystic duct [3]. While intraoperative cholangiograms do not prevent CBD injuries, their routine use seems to decrease the rate at which they occur [4].

One of the most feared complications of preforming a laparoscopic cholecystectomy is a bile duct injury. Intraoperative cholangiography has been shown to minimize the occurrences of bile duct injuries. Biliary tract abnormalities are encountered in $18-39 \%$ of cholecystectomies. While only $3-6 \%$ of those variants predispose to biliary tract injuries [5].

Possible variations include: low insertion of cystic duct, high insertion of the cystic duct, short cystic duct, long cystic duct, cystic duct joining CHD on the left side, and insertion of the cystic duct into either the left or right hepatic duct. Aberrant or accessary right hepatic ducts are considered the most dangerous anatomical variation in the biliary tree as they are not commonly detected by intraoperative cholangiography [3, 4]. There is an estimated one bile duct injury for every 300 cholecystectomies [3].

Intraoperative cholangiograms are extremely safe, as they are performed prior to dissection and should be routinely done on all patients undergoing cholecystectomy [6].

\section{Materials and Methods}

Data were collected from 22 patients undergoing elective cholecystectomy for acute cholecystitis at a tertiary hospital in Houston, Texas. Patients were transferred to the operat- 


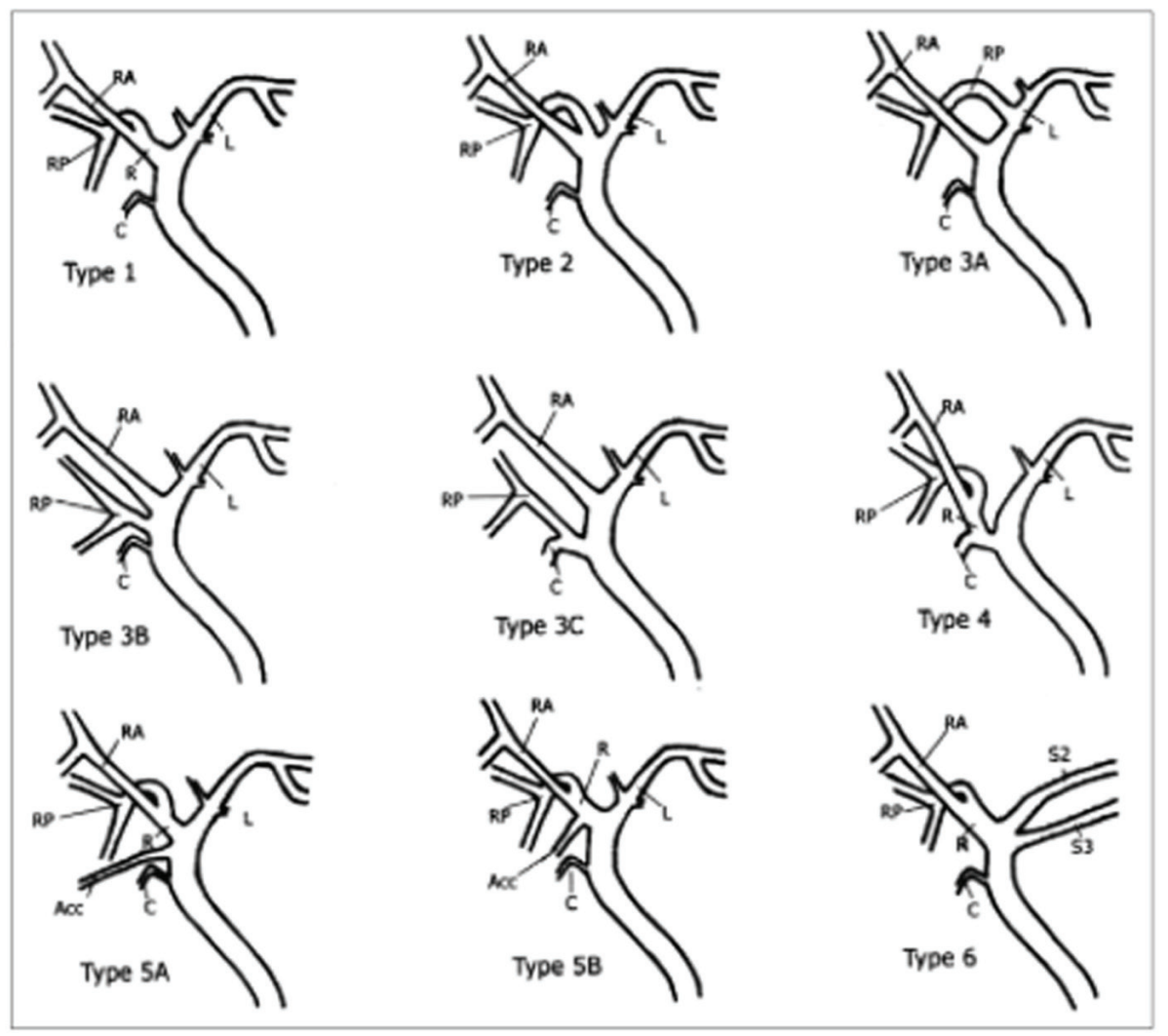

Figure 1. Schematic drawing of IHD anatomy. Type 1 is typical. Type 2 involves triple confluence, the simultaneous emptying of the RASD, RPSD and LHD into the CHD. In type 3, the RPSD drains anomalously, and in type 4, the RHD drains into the cystic duct. In type 5, an accessory duct is present, and in type 6, segments II and III drain individually into the RHD or CHD. Type 7 shows unclassified or complex variation. R: right hepatic duct; L: left hepatic duct; RA: right anterior segmental duct; RP: right posterior segmental duct; C: cystic duct; Acc: accessory duct [7].

ing room and placed in the supine position. All patients received broad-spectrum antibiotics prior to incision. After adequate anesthesia was established, the patients' abdomen was prepped and draped sterilely. A brief time out was performed. Then a small vertical incision was made at the umbilicus and carried through out the layers of the abdominal wall. A Hasson trocar was inserted and pneumoperitoneum was established. Under direct visualization, an $11 \mathrm{~mm}$ non-bladed trocar was placed in the epigastric region followed by two $5 \mathrm{~mm}$ ports in the right upper quadrant. The gallbladder was placed on superior and lateral traction. The structures of the triangle of Calot were then dissected until the critical view of safety was obtained. A clip was placed distally on the cystic duct and a small ductotomy was made. A cholangiogram catheter was inserted through a separate stab incision via an angiocath. This was directed into the cystic duct and an operative cholangiogram was preformed. The cholangiogram catheter was then removed and the cystic duct and artery were divided between the clips. The gallbladder was then dissected off the liver using electrocautery and placed into an endopouch and extracted through the umbilicus. The RUQ was irrigated with saline. Hemostasis was achieved on the gallbladder fossa of the liver with electrocautery. The ports were removed and the gas was evacuated. The umbilical fascial defect was closed with 0 vicryl suture in the figure of eight fashion. The skin incisions were irrigated and closed with subcuticular 4-0 monocryl and dermabond.

The surgeon preforming the procedure at the time read the images obtained from the cholangiogram. Then the images were reviewed at a later time by two separate medical students. Students determined the anatomical variants they read, and then compared their analysis of the cholangiograms to similar variants from other sources (Fig. 1, 2) [1, 7]. If any cholangiogram's read was not unanimously read as the same variation by each of the researchers, the cholangiogram was marked as indeterminate and excluded.

Intraoperative complications including increased bleeding, CBD injury, or any other ductal injury were recorded, in order to compare the risks that present in patients with variants in anatomy.

\section{Results}

Sixty-four percent of patients presented with normal ductal anatomy (Fig. 3). While the remaining $36 \%$ of patients pre- 


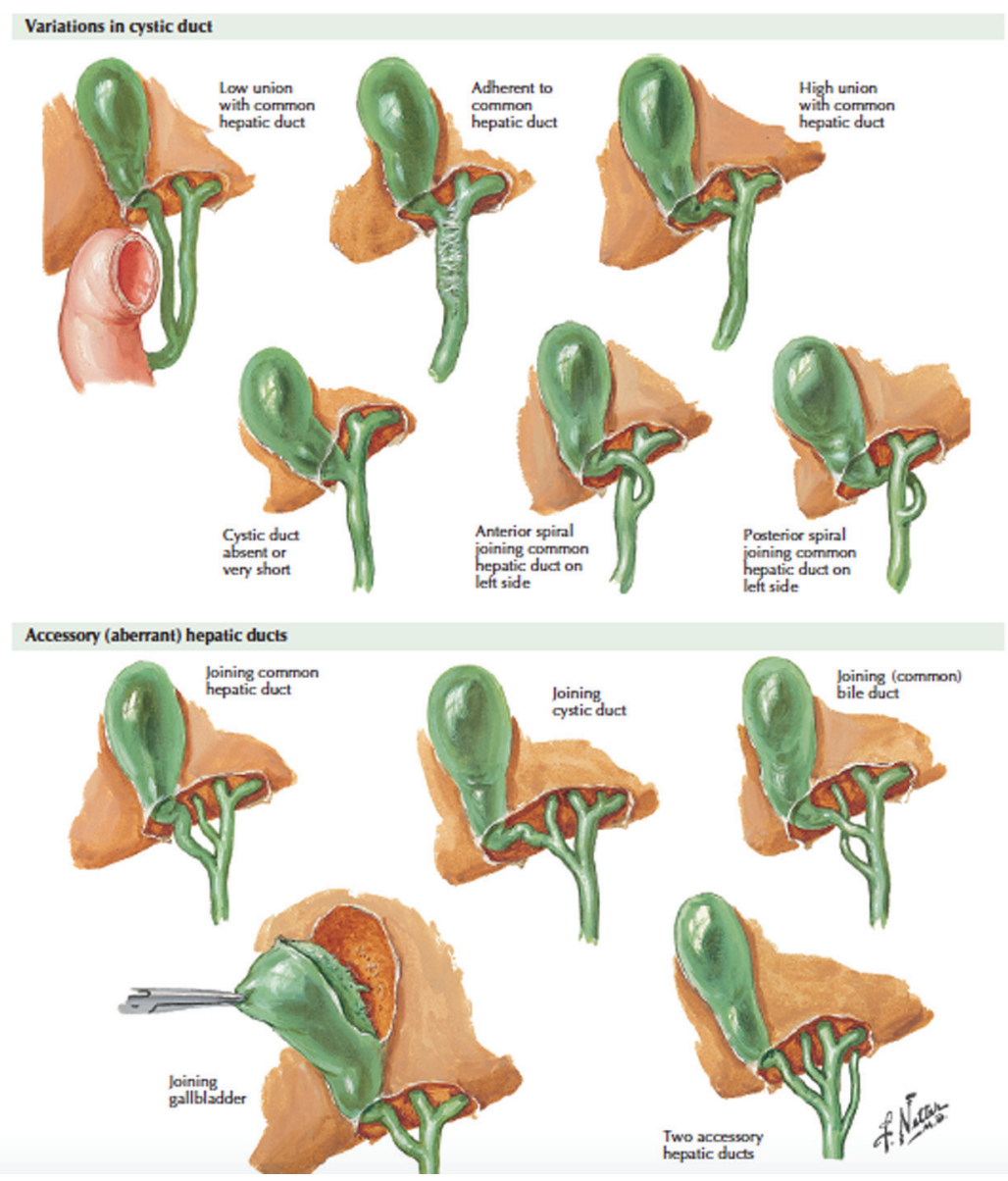

Figure 2. Variations in hepatic and cystic duct [1].

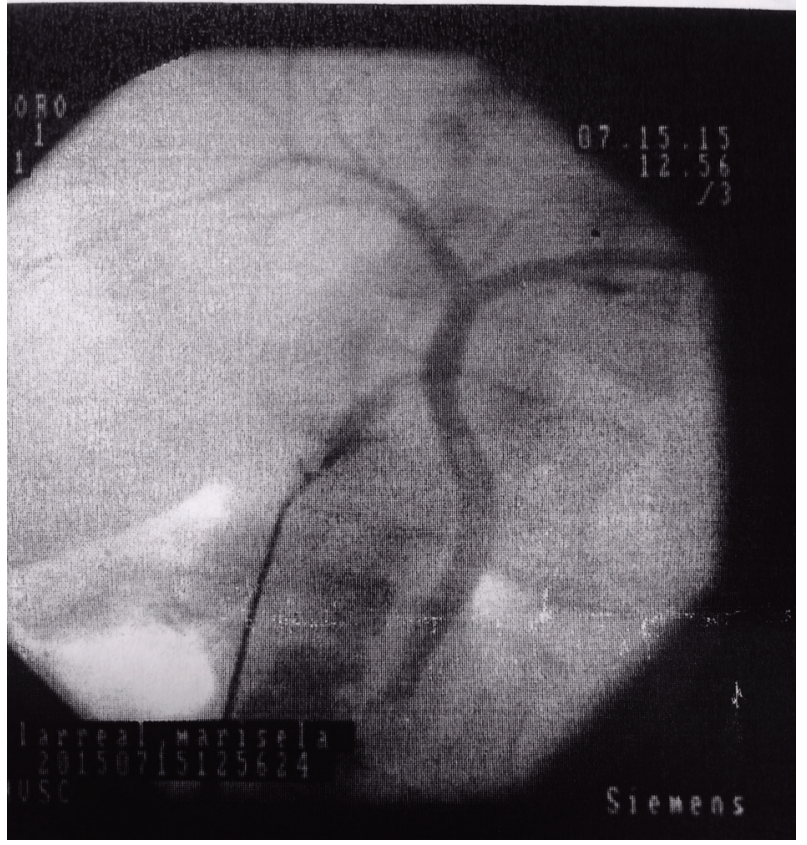

Figure 3. Intraoperative cholangiogram showing normal ductal anatomy.

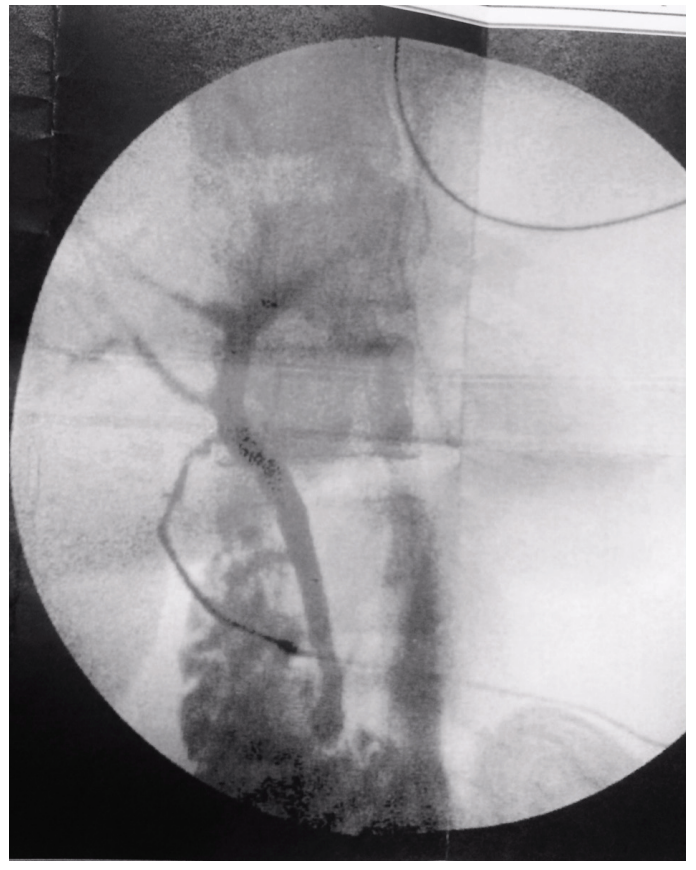

Figure 4. RPSD draining into CHD. 


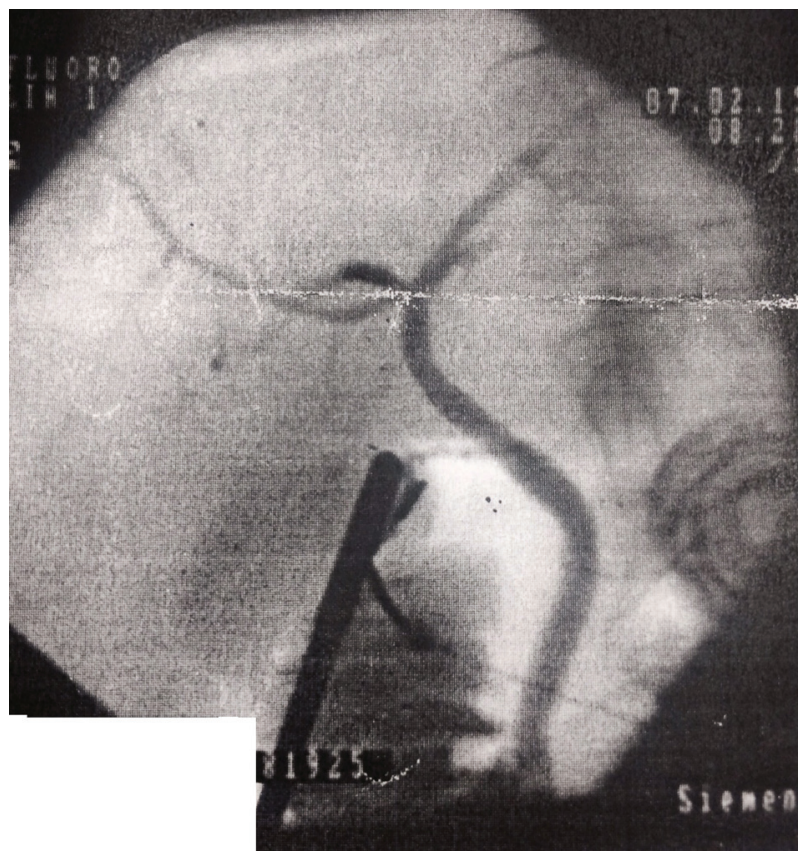

Figure 5. Triple confluence of RASD, RPSD, and LHD into CHD.

sented with anatomic variations in their biliary system, right posterior segmental duct (RPSD) draining into the CBD (Fig. 4), trifurcation of right anterior segmental duct (RASD), RPSD, and left hepatic duct (LHD) draining into the CBD (Fig. 5), and accessory hepatic duct draining into CHD; all occurred at the same frequency of $10.5 \%(n=2)$ each respectively, with the remaining $5.26 \%$ coming from patients with the RPSD draining into the LHD $(\mathrm{n}=1)$ (Table 1). Patients with anatomic variations had zero intraoperative complication. A single patient with normal anatomic structure had an increase of intraoperative blood loss (defined as greater than $100 \mathrm{~mL}$ of blood loss intraoperatively). The blood loss was attributed to the fact that the patient was on blood thinners at the time of surgery (Table 2).

Three cholangiograms were indeterminate and were excluded. Two were concluded to be indeterminate due to unclear imaging and one due to an inconclusive determine in the anatomy by the three researchers.

\section{Discussion}

Accurate understanding of the anatomy at variations is of the utmost importance for a surgeon preforming any surgery. With this being said, variations in the anatomy of the biliary tract have been long recognized. Thus having an accurate understanding of the variants in anatomy is crucial.

Our results faired similar to that of current literature. The most recent data state that $18-39 \%$ of patients have some anatomical variations in their biliary tracts. Our data showed that $36.8 \%(\mathrm{n}=7)$ had unique anatomical variations of the norm. RPSD draining into the CHD $(n=2)$, trifurcation of RASD, RPSD, and LHD draining into the CHD $(\mathrm{n}=2)$, and accessory hepatic duct draining into $\mathrm{CHD}(\mathrm{n}=2)$ all occurred at the same frequency of $10.5 \%$ each respectively. While only $5.26 \%(\mathrm{n}=$ 1) had an RPSD draining into the LHD.

While no patients with variations in ductal anatomy had any adverse complications in our study, it is still of the utmost importance to understand that anatomical variations are frequently present. The most important reason is to prevent CBD injury, which has been shown to be decreased by $50-70 \%$ with the use of intraoperative cholangiography to determine ductal anatomy in previous studies.

Table 1. Number and Type of Anatomic Variants

\begin{tabular}{ll}
\hline Anatomy & Number of patients \\
\hline Normal anatomy & 12 \\
Variants & \\
\hline Accessory hepatic duct draining into CHD & 2 \\
RPSD draining into CHD & 2 \\
Triple confluence of RASD, RPSD and left hepatic duct into CHD & 2 \\
RPSD draining into LHD & 1 \\
\hline
\end{tabular}

Table 2. Complications Associated With Variants

\begin{tabular}{llll}
\hline Anatomy & Increased intraoperative hemorrhage & CBD injury & Other ductal injuries \\
\hline Normal & $1 *$ & 0 & 0 \\
Variants in anatomy & & 0 & 0 \\
$\quad$ Triple confluence of RASD, RPSD, and LHD into CHD & 0 & 0 & 0 \\
Accessory hepatic duct draining into CHD & 0 & 0 & 0 \\
RPSD draining into CHD & 0 & 0 & 0 \\
RPSD draining into LHD & 0 & 0 \\
\hline
\end{tabular}




\section{Acknowledgement}

This project could not have been completed if it was not for the guidance of Michelle Espiritu and Dr. Cameron Ruttman, thank you for your help and support. I would also like to thank my parents, Dr. Amr Elghannam and Helen Elghannam, for their continued support.

\section{Conflict of Interest}

None.

\section{Financial Disclosures}

None.

\section{References}

1. Delaney C, Netter F. Laparoscopic and Open Cholecystectomy. In Netter's surgical anatomy and approaches (pp. 127-140). Philadelphia, PA: Elsevier. 2014.
2. Polat FR, Abci I, Coskun I, Uranues S. The importance of intraoperative cholangiography during laparoscopic cholecystectomy. JSLS. 2000;4(2):103-107.

3. Cameron J, Cameron A. Gallbladder and Biliary tree. In Current surgical therapy: Expert consult (11th ed, pp. 383-427). Philadelphia, PA: Elsevier. 2014.

4. Flum DR, Dellinger EP, Cheadle A, Chan L, Koepsell $\mathrm{T}$. Intraoperative cholangiography and risk of common bile duct injury during cholecystectomy. JAMA. 2003;289(13):1639-1644.

5. Parmeggiani D, Cimmino G, Cerbone D, Avenia N, Ruggero R, Gubitosi A, Docimo G, Mordente S, Misso $\mathrm{C}$, ParmegganiU. Biliary tract injuries during laparoscopic cholecystectomy: Three case reports and literature review. 2010. Http://www.giornalechirurgia.it/ index.php?PAGE $=$ articolo_dett\&id_article $=4013 \& I D$ ISSUE=451, 16-19.

6. Cudjoe EA, Edoga JK, Chattar D. The advantages of preview cholangiography during laparoscopic cholecystectomy. JSLS. 2001;5(3):245-248.

7. Choi JW, Kim TK, Kim KW, Kim AY, Kim PN, Ha HK, Lee MG. Anatomic variation in intrahepatic bile ducts: an analysis of intraoperative cholangiograms in 300 consecutive donors for living donor liver transplantation. Korean J Radiol. 2003;4(2):85-90. 